\title{
Timing of tracheotomy in ICU patients: a systematic review of randomized controlled trials
}

\author{
Koji Hosokawa ${ }^{1 *}$, Masaji Nishimura², Moritoki Egi ${ }^{3}$ and Jean-Louis Vincent ${ }^{1}$
}

\begin{abstract}
Introduction: The optimal timing of tracheotomy in critically ill patients remains a topic of debate. We performed a systematic review to clarify the potential benefits of early versus late tracheotomy.

Methods: We searched PubMed and CENTRAL for randomized controlled trials that compared outcomes in patients managed with early and late tracheotomy. A random-effects meta-analysis, combining data from three a priori-defined categories of timing of tracheotomy (within 4 versus after 10 days, within 4 versus after 5 days, within 10 versus after 10 days), was performed to estimate the weighted mean difference (WMD) or odds ratio (OR).

Results: Of the 142 studies identified in the search, 12, including a total of 2,689 patients, met the inclusion criteria. The tracheotomy rate was significantly higher with early than with late tracheotomy ( $87 \%$ versus $53 \%$, OR 16.1 (5.7-45.7); $\mathrm{p}<0.01$ ). Early tracheotomy was associated with more ventilator-free days (WMD $2.12(0.94,3.30), p<0.01)$, a shorter ICU stay (WMD -5.14 (-9.99, -0.28), $p=0.04)$, a shorter duration of sedation (WMD -5.07 $(-10.03,-0.10), p<0.05)$ and reduced long-term mortality (OR $0.83(0.69-0.99), p=0.04)$ than late tracheotomy.

Conclusions: This updated meta-analysis reveals that early tracheotomy is associated with higher tracheotomy rates and better outcomes, including more ventilator-free days, shorter ICU stays, less sedation, and reduced long-term mortality, compared to late tracheotomy.
\end{abstract}

Keywords: Early tracheotomy, Systematic review, Mortality

\section{Introduction}

Tracheotomy has a number of advantages in patients requiring prolonged mechanical ventilation [1-3], including improved lung mechanics $[4,5]$, easier oral hygiene, diminished nociceptive stimuli on the larynx or trachea, decreased need for sedatives, enhanced communication, and the fact that the head and neck are free of equipment [6-8]. Tracheotomy, however, also has adverse effects, including procedure-related complications and later cosmetic concerns [9-11].

Because of the relatively complex procedure, tracheotomy was in the past reserved for patients who had

\footnotetext{
* Correspondence: khosok@gmail.com

'Department of Intensive Care, Erasme University Hospital, Université Libre de Bruxelles, Route de Lennik 808, 1070 Brussels, Belgium

Full list of author information is available at the end of the article
}

been intubated for a long time [12]. However, technological improvements, including simplification and decreased invasiveness of the procedure, have encouraged some to consider a more liberal use of tracheotomy. Some earlier studies in ICU patients suggested that early tracheotomy was associated with better outcomes than late tracheotomy [13-16], but more recent, rigorously designed randomized controlled trials (RCTs) did not show a significant survival benefit [17-19]. The five most recent systematic reviews of RCTs comparing early and late tracheotomy yielded conflicting results [20-24]. However, these metaanalyses combined studies using different timings of early (within 48 hours [16], within 4 days [19], and between 6 and 8 days [17]) versus late interventions, so that the results were difficult to interpret. A meta- 
analysis in which only studies with early tracheotomy performed within 4 days or 7 days were included reported no significant differences between early and late tracheotomy [24].

Since the most recent systematic reviews were conducted, results from an RCT by Diaz-Prieto et al., which included about 500 patients, have been published [25]. We therefore conducted an updated systematic review and meta-analysis to evaluate the impact of early tracheotomy compared to late tracheotomy on outcome. To investigate whether very early (within 4 days) tracheotomy has a greater impact on outcome than relatively early (within 10 days) tracheotomy, we also evaluated possible differences between very early, relatively early and late tracheotomy.

\section{Methods}

This systematic review was conducted according to the preferred reporting items for systematic reviews and meta-analyses (PRISMA) statement [26].

Two authors (KH and ME) searched PubMed and the Cochrane Central Register of Controlled Trials (CENTRAL) on 3 July 2015 using relevant terms (Additional file 1: Table S1). They also independently examined the reference lists from related articles or systematic reviews. Disagreements about eligibility were resolved by consensus. Articles eligible for inclusion were RCTs that compared outcomes associated with early and late tracheotomy. The definition of timing was not specified in the selection step. Studies on pediatric patients, reviews, conference abstracts, quasi-randomized prospective trials and non-Englishlanguage articles were excluded. The quality of studies was examined using the method recommended by a Cochrane Collaboration tool for assessing risk of bias in the included RCTs [27].

From the included articles, two of the authors (KH and ME) extracted timing of tracheotomy, number of participating centers, type and number of ICUs, number of patients and the inclusion and exclusion criteria, major disease categories, severity scores, the rate of tracheotomy, the rate of percutaneous dilatation procedures, duration of mechanical ventilation and/or ventilator-free days (VFDs), duration of ICU stay and/or ICU-free days, duration of sedation and/ or sedation-free days, the rate of acquired pneumonia, and short-term (defined a priori as $\leq 2$ months) and long-term (defined a priori as $>2$ months and in hospital) term mortality rates. We also recorded complication rates related to tracheotomy and unplanned extubation. No attempts were made to contact the authors of included studies to obtain missing/unreported data.
Meta-analysis was performed using Review Manager (ver. 5.3, The Nordic Cochrane Center, Copenhagen, Denmark). When continuous values were described by median and IQR or range instead of mean and SD, the following formula was used for approximations:

Mean $=$ Median; $\mathrm{SD}=\mathrm{IQR} / 1.35 ;$ if $15<\mathrm{n}<70, \mathrm{SD}=$ (b-a) $/ 4$, (Minimum (a), Maximum (b)); if $\mathrm{n}>70, \mathrm{SD}=$ (b-a)/6 [27, 28].

All pooled data were assessed using a random-effects model with an inverse variance method. The estimation of combined continuous values and dichotomous values was described by weighted mean difference (WMD) or odds ratios (OR), respectively, with $95 \%$ CI. We first performed analysis by dividing the data into three groups of studies defined a priori according to the definitions used by the original articles for early versus late timings (within 4 versus after 10 days, within 4 versus after 5 days, or within 10 versus after 10 days) and then combined the results to give an overall estimation of early versus late tracheotomy. Heterogeneity among the included studies was assessed using $\mathrm{Tau}^{2}, \mathrm{Chi}^{2}$ and $I^{2}$ tests. A $p$ value $<0.05$ was considered significant.

\section{Results}

Among 142 citations initially identified, 34 studies were selected for full-text reading (see Additional file 1: Fig. S1). Of these, 13 studies were excluded because they were systematic reviews. Nine others were excluded because of unclear inclusion criteria [29], inadequate randomization $[30,31]$, randomization at different timings and re-allocation to different groups [32], missing patient data before randomization [33, 34], inadequate outcome assessment [35], and non-Englishlanguage articles [36, 37]. A total of 12 eligible RCTs $[16-19,25,38-44]$ including 2,689 patients were therefore included (Additional file 1: Fig. S1). The studies were similar in terms of quality assessment (Additional file 1: Fig. S2).

The definitions of early and late tracheotomy varied among the studies (Table 1). Seven studies used very early tracheotomy (within 4 days) [16, 19, 38, 40-43] and five used early tracheotomy (within 10 days) [17, 18, $25,39,44]$. Late tracheotomy was defined as after 10 days in 10 RCTs $[16-19,25,38-41,44]$ and as after 5 days in 2 studies $[42,43]$. The studies included different patient populations, including patients with intracranial disease [43], trauma [39], burns [38], and postoperative patients $[18,41,42]$ (Table 1). Some studies excluded patients with pneumonia [17, 41, 42, 44]. Tracheotomy was performed primarily using percutaneous methods in 9 of the 11 studies $[16-19,25,41-44]$ that provided this information (Table 1). The reported incidence of complications 
Table 1 Summary of the included randomized controlled trials of early versus late tracheotomy

\begin{tabular}{|c|c|c|c|c|c|c|c|c|c|}
\hline \multirow[t]{2}{*}{ Study } & \multirow[b]{2}{*}{$\begin{array}{l}\text { Definition of } \\
\text { early versus late } \\
\text { tracheotomy } \\
\text { (days) }\end{array}$} & \multirow[b]{2}{*}{$\begin{array}{l}\text { Type of ICU; } \\
\text { number of } \\
\text { ICUs }\end{array}$} & \multirow[b]{2}{*}{$\begin{array}{l}\text { Number of } \\
\text { patients, early } \\
\text { versus late } \\
\text { groups }\end{array}$} & \multirow[b]{2}{*}{ Inclusion criteria } & \multirow[b]{2}{*}{ Excluded } & \multicolumn{2}{|l|}{ Patients } & \multirow[b]{2}{*}{$\begin{array}{l}\text { Tracheotomy rate } \\
\text { (number }(\%)) \text { in } \\
\text { early versus late } \\
\text { groups }\end{array}$} & \multirow[b]{2}{*}{$\begin{array}{l}\text { Percutaneous dilatation } \\
\text { tracheotomy (number } \\
\text { (\%)) in early versus } \\
\text { late groups }\end{array}$} \\
\hline & & & & & & $\begin{array}{l}\text { Major disease } \\
\text { category }\end{array}$ & $\begin{array}{l}\text { APACHE } \\
\text { \|/SAPS \| }\end{array}$ & & \\
\hline Saffle et al. (2002) [38] & $2-4$ vs. $14-16$ & Burn; 1 & 21 vs. 23 & $\begin{array}{l}\text { High predicted } \\
\text { probability of } \\
\text { prolonged MV }\end{array}$ & & Burn (100\%) & NA & $\begin{array}{l}21(100 \%) \text { vs. } \\
16(70 \%)\end{array}$ & NA \\
\hline Rumbak et al. (2004) [16] & $\leq 2$ vs. $>14$ & Medical; 2 & 60 vs. 60 & $\begin{array}{l}\text { exp. }>14 \text { d MV; } \\
\text { APACHE II > } 25\end{array}$ & & $\begin{array}{l}\text { Respiratory failure } \\
(100 \%) \text {, severe } \\
\text { sepsis }(68 \%)\end{array}$ & 26.9 & $\begin{array}{l}60(100 \%) \text { vs. } \\
50(83 \%)\end{array}$ & All in both groups \\
\hline Barquist et al. (2006) [39] & $<8$ vs. $>28$ & Trauma; 1 & 29 vs. 31 & $\begin{array}{l}\text { GCS }>4 \text { with } \\
\text { no head injury; } \\
\text { GCS }>9 \text { with } \\
\text { head injury }\end{array}$ & & Trauma (100 \%) & 12.6 & $\begin{array}{l}27(93 \%) \text { vs. } \\
11(35 \%)\end{array}$ & $\begin{array}{l}0 / 27 \text { (0 \%) vs. 0/11 } \\
(0 \%)\end{array}$ \\
\hline Blot et al. (2008) [40] & $\leq 4$ vs. $>14$ & $\begin{array}{l}\text { Medical and } \\
\text { surgical; } 25\end{array}$ & 61 vs. 62 & exp. $>7$ d MV & $\begin{array}{l}\text { Irreversible } \\
\text { neurological } \\
\text { disease }\end{array}$ & $\begin{array}{l}\text { Respiratory failure } \\
(33 \%) \text {, neurology } \\
(23 \%) \text {, trauma } \\
(19 \%)\end{array}$ & $\mathrm{NA} / 50$ & $\begin{array}{l}60(98 \%) \text { vs. } \\
16(26 \%)\end{array}$ & $\begin{array}{l}19 / 60(32 \%) \text { vs. } \\
7 / 16(44 \%)\end{array}$ \\
\hline Terragni et al. (2010) [17] & 6-8 vs. $13-15$ & $\mathrm{NA} ; 12$ & 209 vs. 210 & $\begin{array}{l}\text { SAPS } \|=35-65 ; \\
\text { SOFA } \geq 5 \text {; worsening } \\
\text { respiratory conditions; } \\
\text { unchanged/worse } \\
\text { SOFA sore }\end{array}$ & $\begin{array}{l}\text { Pneumonia } \\
\text { (CPIS } \geq 6 \text { ) } \\
\text { COPD }\end{array}$ & $\begin{array}{l}\text { Respiratory } \\
\text { failure (46\%), } \\
\text { neurology (24 \%), } \\
\text { cardiovascular } \\
\text { disease (23\%) }\end{array}$ & NA/50.4 & $\begin{array}{l}145 \text { (69 \%) vs. } \\
119(57 \%)\end{array}$ & $\begin{array}{l}141 / 145 \text { (97 \%) vs. } \\
113 / 119(95 \%)\end{array}$ \\
\hline Trouillet et al. (2011) [18] & $<5-7$ vs. $>19$ & Surgical; 1 & 109 vs. 107 & exp. $>7$ d MV & $\begin{array}{l}\text { Irreversible } \\
\text { neurologic } \\
\text { disorder }\end{array}$ & $\begin{array}{l}\text { Post-cardiac surgery } \\
(100 \%)\end{array}$ & NA/46.5 & $\begin{array}{l}109(100 \%) \text { vs. } \\
29(27 \%)\end{array}$ & All in both groups \\
\hline Zheng et al. (2012) [41] & 3 vs. 15 & Surgical; 1 & 58 vs. 61 & $\begin{array}{l}\mathrm{PaO}_{2} / \mathrm{FiO}_{2}<200 ; \\
\text { APACHE II >15; } \\
\text { SOFA > } 5 \text {; CPIS } \\
>6 \text {; exp. >14 d MV }\end{array}$ & $\begin{array}{l}\text { Pulmonary } \\
\text { infection } \\
(C P I S>6)\end{array}$ & NA & 20.0 & $\begin{array}{l}58(100 \%) \text { vs. } \\
51(84 \%)\end{array}$ & All in both groups \\
\hline Koch et al. (2012) [42] & $\leq 4$ vs. $\geq 6$ & Surgical; 1 & 50 vs. 50 & exp. $>21 \mathrm{~d}$ MV & Pneumonia & $\begin{array}{l}\text { Neurosurgical } \\
(28 \%) \text {, trauma } \\
(25 \%)\end{array}$ & 22 & $\begin{array}{l}\text { All in both } \\
\text { groups }\end{array}$ & All in both groups \\
\hline Young et al. (2013) [19] & $\leq 4$ vs. $>10$ & $\begin{array}{l}\text { General; } 70 \text { and } \\
\text { surgical; } 2\end{array}$ & 451 vs. 448 & exp. $>7$ d MV & $\begin{array}{l}\text { Respiratory } \\
\text { failure due } \\
\text { to chronic } \\
\text { neurological } \\
\text { disease }\end{array}$ & $\begin{array}{l}\text { Pulmonary }(60 \%) \text {, } \\
\text { gastrointestinal } \\
(19 \%)\end{array}$ & 19.8 & $\begin{array}{l}418(93 \%) \text { vs. } \\
204(46 \%)\end{array}$ & $\begin{array}{l}378 / 418(90 \%) \text { vs. } \\
176 / 204(86 \%)\end{array}$ \\
\hline Bösel et al. (2013) [43] & $\leq 3$ vs. $7-14$ & Neuro; 1 & 30 vs. 30 & $\begin{array}{l}\text { ICH; SAH; or AIS; } \\
\text { exp. >14 d MV }\end{array}$ & $\begin{array}{l}\text { Severe chronic } \\
\text { cardiopulmonary } \\
\text { disease; extensive } \\
\text { brainstem lesions }\end{array}$ & $\begin{array}{l}\text { Non-traumatic } \\
\text { neurology (100 \%) }\end{array}$ & 17 & $\begin{array}{l}30(100 \%) \text { vs. } \\
18(60 \%)\end{array}$ & $\begin{array}{l}27 / 30(90 \%) \text { vs. } \\
16 / 18(89 \%)\end{array}$ \\
\hline
\end{tabular}


Table 1 Summary of the included randomized controlled trials of early versus late tracheotomy (Continued)

\begin{tabular}{|c|c|c|c|c|c|c|c|c|c|}
\hline Mohamed et al. (2014) [44] & $\leq 10$ vs. $>10$ & $N A ; 2$ & 20 vs. 20 & APACHE $\geq 15$ & Pneumonia & $\begin{array}{l}\text { TBI (43 \%), CVA } \\
(25 \%)\end{array}$ & 24 & $\begin{array}{l}\text { All in both } \\
\text { groups }\end{array}$ & All in both groups \\
\hline Diaz-Prieto et al. (2014) [25] & $<8$ vs. $>14$ & NA; 4 & 245 vs. 244 & $\begin{array}{l}\text { 1, exp. }>7 \text { d MV; } 2 \\
\text { attending physician's } \\
\text { acceptance at 3-5 d }\end{array}$ & & $\begin{array}{l}\text { Respiratory } \\
\text { insufficiency (60 \%), } \\
\text { coma ( } 22 \%)\end{array}$ & 20 & $\begin{array}{l}167 \text { (68 \%) vs. } \\
135(55 \%)\end{array}$ & All in both groups \\
\hline
\end{tabular}

Values are shown as days from the initiation of mechanical ventilation, except one that used days from ICU admission [19]. AIS acute ischemic stroke, APACHE acute physiology and chronic health evaluation, COPD chronic obstructive pulmonary disease, CPIS clinical pulmonary infection score, CVA cerebrovascular accident, $d$ days, exp. expected, GCS Glasgow coma scale, ICH intracerebral hemorrhage, MV mechanical ventilation , $N A$ not available, $\mathrm{PaO}_{2} / \mathrm{FiO}_{2}$ partial pressure arterial oxygen/fraction of inspired oxygen, $R C T$ randomized controlled trial, SAH subarachnoid hemorrhage, SAPS simplified acute physiology score, SOFA sequential organ failure assessment 
related to tracheotomy ranged from $0 \%$ to $39 \%$, with the most frequent reported complication being bleeding (data not shown).

\section{Meta-analysis results \\ Tracheotomy rate}

The rate of tracheotomy was significantly higher with early than with late tracheotomy in studies comparing timings of within 4 versus after 10 days (95\% versus $52 \%$, OR 24.08) and in those comparing within 10 versus after 10 days (76 \% versus $51 \%$, OR 5.32, Fig. 1). When the data were combined for the 12 studies $[16-19,25,38-44]$, the rates were $87 \%$ for early versus $53 \%$ for late tracheotomy (OR 16.12 (5.68, 45.74), $p<0.01 ; \mathrm{I}^{2} 92 \%, p$ heterogeneity $\left.<0.01\right)$.

\section{Mechanical ventilation}

The duration of mechanical ventilation was reported in eight studies [16, 18, 19, 38, 40, 42-44] (Table 2) and did not differ significantly between the early and late tracheotomy groups in any of the three predefined groups of studies or overall (Fig. 2a). VFDs were reported in five studies [17, 18, 25, 39, 41] (Table 2) and were greater with early than with late tracheotomy in one of the predefined groups of studies (within 10 versus after 10 days; WMD $2.10(0.44,3.76), p<0.01 ; I^{2}$ $55 \%, p$ heterogeneity $=0.09$; Fig. $2 \mathrm{~b})$ and overall (WMD $2.12(0.94,3.30), p<0.01 ; I^{2} 40 \%, p$ heterogeneity $=0.16$; Fig. $2 b$ ).

\section{ICU stay}

The duration of ICU stay was reported in seven studies [16, 18, 19, 25, 42-44] (Table 2) and was significantly shorter with early than with late tracheotomy overall (WMD $-5.14(-9.99,-0.28), p=0.04 ; I^{2}$ $96 \%, p$ heterogeneity <0.01; Additional file 1: Figure S3a). Three studies reported ICU-free days [17, 39, 41]: there were no significant differences with early compared to late tracheotomy overall (Additional file 1: Figure S3b).

\section{Sedation}

The duration of sedation was reported in four studies $[16,18,19,25]$ (Table 2) and was shorter with early than with late tracheotomy in one of the predefined groups of studies (within 10 versus after 10 days) and overall (WMD -5.07 (-10.03, -0.10), $p<0.05 ; I^{2} 99 \%, p$ heterogeneity <0.01; Fig. 3a). The number of sedation-free days was reported in three studies [18, 40,41] and was larger with early than with late tracheotomy in two of the predefined groups of studies (within 4 versus after 10 days, and within 10 versus after 10 days) and overall (WMD $3.68(2.93,4.44), p<0.01 ; I^{2} 0 \%, p$ heterogeneity $=0.82$; Fig. 3b).

\section{Acquired pneumonia}

The risk of acquired pneumonia was reported in 10 studies $[16-18,25,38-42,44]$ (Table 2) and did 
Table 2 Reported outcomes in the included randomized controlled trials

\begin{tabular}{|c|c|c|c|c|c|c|c|c|c|c|}
\hline & $\begin{array}{l}\text { Duration of } \\
\text { mechanical } \\
\text { ventilation, early } \\
\text { versus late } \\
\text { groups (days) }\end{array}$ & $\begin{array}{l}\text { Number of } \\
\text { ventilator-free } \\
\text { days in } 28 \text { days, } \\
\text { early versus } \\
\text { late groups }\end{array}$ & $\begin{array}{l}\text { Duration of } \\
\text { ICU stay, early } \\
\text { versus late } \\
\text { groups (days) }\end{array}$ & $\begin{array}{l}\text { Number of } \\
\text { ICU-free days } \\
\text { in } 28 \text { days, } \\
\text { early versus } \\
\text { late groups }\end{array}$ & $\begin{array}{l}\text { Duration of } \\
\text { sedation, early } \\
\text { versus late } \\
\text { groups (days) }\end{array}$ & $\begin{array}{l}\text { Number of } \\
\text { sedation-free } \\
\text { days in } 28 \text { days, } \\
\text { early versus late } \\
\text { groups }\end{array}$ & $\begin{array}{l}\text { Acquired } \\
\text { pneumonia, } \\
\text { early versus } \\
\text { late groups }\end{array}$ & $\begin{array}{l}\text { Mortality } \\
\text { ( } \leq 2 \text { months), } \\
\text { early versus } \\
\text { late groups }\end{array}$ & $\begin{array}{l}\text { Mortality } \\
\text { (>2 months), } \\
\text { early versus } \\
\text { late groups }\end{array}$ & $\begin{array}{l}\text { Other outcomes, } \\
\text { early versus late } \\
\text { groups, }\end{array}$ \\
\hline $\begin{array}{l}\text { Saffle et al. } \\
\text { (2002) [38] }\end{array}$ & $\begin{array}{l}35.5(4.5) \text { vs. } \\
31.4(5.2) \\
(p, N A)\end{array}$ & NA & NA & NA & NA & NA & $\begin{array}{l}21(100 \%) \text { vs. } \\
22(96 \%) \\
(p=0.16)\end{array}$ & $\begin{array}{l}17(81 \%) \text { vs. } \\
17(74 \%) \\
(p=0.58)\end{array}$ & NA & $\begin{array}{l}\text { Successfully } \\
\text { extubated, } \\
1(5 \%) \text { vs. } \\
6(26 \%) \\
(p<0.01)\end{array}$ \\
\hline $\begin{array}{l}\text { Rumbak et al. } \\
\text { (2004) [16] }\end{array}$ & $\begin{array}{l}7.6(4.0) \text { vs. } \\
17.4(5.3) \\
(p<0.01)\end{array}$ & NA & $\begin{array}{l}4.8(1.4) \text { vs. } \\
16.2(3.8) \\
(p<0.01)\end{array}$ & NA & $\begin{array}{l}3.2(0.4) \text { vs. } \\
14.1(2.9) \\
(p<0.01)\end{array}$ & NA & $\begin{array}{l}3(5 \%) \text { vs. } \\
15(25 \%) \\
(p<0.05)\end{array}$ & $\begin{array}{l}19(32 \%) \text { vs, } \\
37(62 \%) \\
(p<0.05) \\
\text { (at } 30 \text { d) }\end{array}$ & NA & $\begin{array}{l}\text { Damage to the } \\
\text { larynx and lips, } \\
\text { rated } 0-1 \text { vs. } \\
2-3\end{array}$ \\
\hline $\begin{array}{l}\text { Barquist et al. } \\
\text { (2006) [39] }\end{array}$ & NA & $\begin{array}{l}8.57(7.9) \text { vs. } \\
8.83(9) \\
\text { (in } 30 \mathrm{~d}) \\
(p=0.9)\end{array}$ & NA & $\begin{array}{l}5.0(6.0) \text { vs. } \\
5.3(6.5) \\
\text { (in } 30 \mathrm{~d}) \\
(p=0.8)\end{array}$ & NA & NA & $\begin{array}{l}28(97 \%) \text { vs. } \\
28(90 \%) \\
(p=0.6)\end{array}$ & $\begin{array}{l}2(6.9 \%) \text { vs. } \\
5(16 \%) \\
(p=0.4)\end{array}$ & NA & \\
\hline $\begin{array}{l}\text { Blot et al. } \\
\text { (2008) [40] }\end{array}$ & $\begin{array}{l}14(2-28) \text { vs. } \\
16(3-28) \\
(p=0.62)\end{array}$ & NA & NA & NA & NA & $\begin{array}{l}18(0-27) \text { vs. } \\
15(0-27)\end{array}$ & $\begin{array}{l}30(49 \%) \text { vs. } \\
31(50 \%) \\
(p=0.94)\end{array}$ & $\begin{array}{l}12(20 \%) \text { vs. } \\
15(24 \%) \\
\text { (at } 28 \mathrm{~d}) ; 16 \\
(27 \%) \text { vs. } 15 \\
(24 \%)(\text { at } \\
60 \text { d) }\end{array}$ & NA & $\begin{array}{l}\text { Laryngeal } \\
\text { symptoms, } \\
1(2 \%) \text { vs. } \\
7(11 \%) \\
(p=0.01)\end{array}$ \\
\hline $\begin{array}{l}\text { Terragni et al. } \\
\text { (2010) [17] }\end{array}$ & NA & $\begin{array}{l}11(0-21) \text { vs. } \\
6(0-17) \\
(p=0.02)\end{array}$ & NA & $\begin{array}{l}0(0-13) \text { vs. } \\
0(0-8) \\
(p=0.02)\end{array}$ & NA & NA & $\begin{array}{l}30(14 \%) \text { vs. } \\
44(21 \%) \\
(p=0.07)\end{array}$ & $\begin{array}{l}55(26 \%) \text { vs. } \\
66(31 \%) \\
(p=0.25) \\
\text { (at } 28 d)\end{array}$ & $\begin{array}{l}72 / 144(50 \%) \\
\text { vs. } 75 / 138 \\
(57 \%)(p=0.25) \\
\text { (in } 1 \text { year) }\end{array}$ & $\begin{array}{l}\text { Successful } \\
\text { weaning, } \\
161(77 \%) \\
\text { vs. } 142(68) \\
(p=0.02)\end{array}$ \\
\hline $\begin{array}{l}\text { Trouillet et al. } \\
\text { (2011) [18] }\end{array}$ & $\begin{array}{l}17.9(14.9) \text { vs. } \\
19.3(16.9) \\
(p=0.55)\end{array}$ & $\begin{array}{l}10.0(8.8) \text { vs. } \\
9.2(10.2) \\
(p=0.52)\end{array}$ & $\begin{array}{l}23.9(21.3) \text { vs. } \\
25.5(22.2) \\
(p=0.85)\end{array}$ & NA & $\begin{array}{l}6.4(5.9) \text { vs. } 9.6 \\
(7.3)(p<0.01)\end{array}$ & $\begin{array}{l}19.0(9.1) \text { vs. } \\
15.5(9.3) \\
(p<0.01)\end{array}$ & $\begin{array}{l}50(46 \%) \text { vs. } \\
47(44 \%) \\
(p=0.77)\end{array}$ & $\begin{array}{l}17(16 \%) \text { vs. } \\
23(21 \%) \\
(p=0.30) \\
(\text { at } 30 \mathrm{~d})\end{array}$ & $\begin{array}{l}12 / 74(16 \%) \text { vs. } \\
17 / 74(23 \%) \\
(p=0.49) \\
\text { (in } 2.4 \text { years in } \\
\text { mean) }\end{array}$ & $\begin{array}{l}\text { ADL, anxiety, } \\
\text { depression, } \\
\text { or PTSD, } \\
\text { similar }\end{array}$ \\
\hline $\begin{array}{l}\text { Zheng et al. } \\
\text { (2012) [41] }\end{array}$ & NA & $\begin{array}{l}9.6(5.6) \text { vs. } \\
7.4(6.2) \\
(p=0.05)\end{array}$ & NA & $\begin{array}{l}8.0 \\
(5.0-12.0) \text { vs. } \\
3.0(0-12.0) \\
(p<0.01)\end{array}$ & NA & $\begin{array}{l}20.8(2.4) \text { vs. } \\
17.1(2.3) \\
(p=0.05)\end{array}$ & $\begin{array}{l}17(29 \%) \text { vs. } \\
30(49 \%) \\
(p=0.03)\end{array}$ & $\begin{array}{l}8(14 \%) \text { vs. } \\
6(10 \%) \\
(p=0.55) \\
\text { (at } 28 \mathrm{~d})\end{array}$ & NA & \\
\hline $\begin{array}{l}\text { Koch et al. } \\
\text { (2012) [42] }\end{array}$ & $\begin{array}{l}15.3(9.1-19.8) \\
\text { vs. } 21.1 \\
(13.5-27.9) \\
(p \leq 0.01)\end{array}$ & NA & $\begin{array}{l}21.5(15.0-30.0) \\
\text { vs. } 30.6(22.0-37.0) \\
(p \leq 0.05)\end{array}$ & NA & NA & NA & $\begin{array}{l}19(38 \%) \text { vs. } \\
32(64 \%)\end{array}$ & $\begin{array}{l}9(18 \%) \text { vs. } \\
7(14 \%) \\
(p=0.79) \\
\text { (in ICU) }\end{array}$ & $\begin{array}{l}10(20 \%) \text { vs. } \\
11(22 \%)(p=0.81) \\
\text { (in hospital) }\end{array}$ & \\
\hline $\begin{array}{l}\text { Young et al. } \\
\text { (2013) [19] }\end{array}$ & $\begin{array}{l}13.6(12.0) \text { vs. } \\
15.2(14.4) \\
(p=0.06)\end{array}$ & NA & $\begin{array}{l}13.0(8.2-19.1) \\
\text { vs. } 13.1(7.4-23.6) \\
(p=0.74) \text { in } \\
\text { survivors; } 9.3 \\
(4.2-16.0) \text { vs. }\end{array}$ & NA & $\begin{array}{l}5(3-9) \text { vs. } \\
8(4-12) \\
(p<0.01) \text { in } \\
\text { survivors; } 5 \\
(3-9) \text { vs. } 6(4-10)\end{array}$ & NA & NA & $\begin{array}{l}139(31 \%) \\
\text { vs. } 141 \\
(32 \%) \\
(p=0.89) \\
\text { (at } 30 \text { d) }\end{array}$ & $\begin{array}{l}168 \text { (40 \%) vs. } 180 \\
(41 \%)(p=0.63) \text { (in } \\
\text { hospital); } 207 \text { (46\%) } \\
\text { vs. } 217(49 \%) \\
(p=0.38) \text { (1 year) }\end{array}$ & $\begin{array}{l}\text { Antibiotic use, } \\
5(1-8) \text { vs. } \\
5(1-10) \\
(p=0.95) \\
\text { (in } 30 \text { d) }\end{array}$ \\
\hline
\end{tabular}


Table 2 Reported outcomes in the included randomized controlled trials (Continued)

\begin{tabular}{|c|c|c|c|c|c|c|c|c|c|c|}
\hline & & & $\begin{array}{l}10.4(6.0-19.7) \\
(p=0.16) \text { in } \\
\text { non-survivors }\end{array}$ & & $\begin{array}{l}(p=0.11) \text { in } \\
\text { non-survivors }\end{array}$ & & & & & \\
\hline $\begin{array}{l}\text { Bösel et al. } \\
\text { (2013) [43] }\end{array}$ & $\begin{array}{l}15(10-17) \text { vs. } \\
12(8-16) \\
(p=0.23)\end{array}$ & NA & $\begin{array}{l}17(13-22) \text { vs. } \\
18(16-28) \\
(p=0.38)\end{array}$ & NA & NA & NA & NA & $\begin{array}{l}3(10 \%) \text { vs. } \\
14(47 \%) \\
(p<0.01) \\
\text { (in ICU) }\end{array}$ & $\begin{array}{l}8(27 \%) \text { vs. } 18 \\
\text { (0.6\%) ( } p=0.02) \\
\text { (in } 6 \text { months) }\end{array}$ & $\begin{array}{l}\text { Sedation use } \\
(42 \%) \text { vs. } \\
(62 \%)(p=0.02)\end{array}$ \\
\hline $\begin{array}{l}\text { Mohamed et al. } \\
\text { (2014) [44] }\end{array}$ & $\begin{array}{l}20.6(13.0) \text { vs. } \\
32.2(10.5) \\
(p<0.01)\end{array}$ & NA & $\begin{array}{l}21.1(13.5) \text { vs. } \\
40.2(12.7) \\
(p<0.01)\end{array}$ & NA & NA & NA & $\begin{array}{l}4(20 \%) \text { vs. } \\
8(40 \%)\end{array}$ & NA & $\begin{array}{l}8(40 \%) \text { vs. } 8 \\
(40 \%) \text { (in } \\
\text { hospital) }\end{array}$ & \\
\hline $\begin{array}{l}\text { Diaz-Prieto et al. } \\
\text { (2014) [25] }\end{array}$ & NA & $\begin{array}{l}11(0-22) \text { vs. } \\
9(0-22) \\
(p=0.05)\end{array}$ & $\begin{array}{l}22(6-101) \\
22.5(6-174) \\
(p=0.31)\end{array}$ & NA & $\begin{array}{l}11(2-92) \text { vs. } \\
14(0-79) \\
(p=0.02)\end{array}$ & NA & $\begin{array}{l}33(13 \%) \text { vs. } \\
23(9 \%) \\
(p=0.16)\end{array}$ & $\begin{array}{l}42(17 \%) \text { vs. } \\
47(19 \%) \\
(p=0.54) \\
\text { (at } 28 d)\end{array}$ & $\begin{array}{l}63(26 \%) \text { vs. } 73 \\
(30 \%)(p=0.30) \\
\text { (at } 90 \text { d); } 67 \\
(27 \%) \text { vs. } 78 \\
(32 \%)(p=0.26) \\
\text { (in hospital) }\end{array}$ & $\begin{array}{l}\text { Excluded by } \\
\text { attending } \\
\text { physician, } 284 \\
(58 \%)\end{array}$ \\
\hline
\end{tabular}

The values are presented as number (\%), mean with (SD) or median with (IOR). The values indicate early tracheostomy versus late tracheostomy

$A D L$ activities of daily living, $d$ days, NA not available, PTSD posttraumatic stress disorder, RCT randomized controlled trial 


\section{a)}

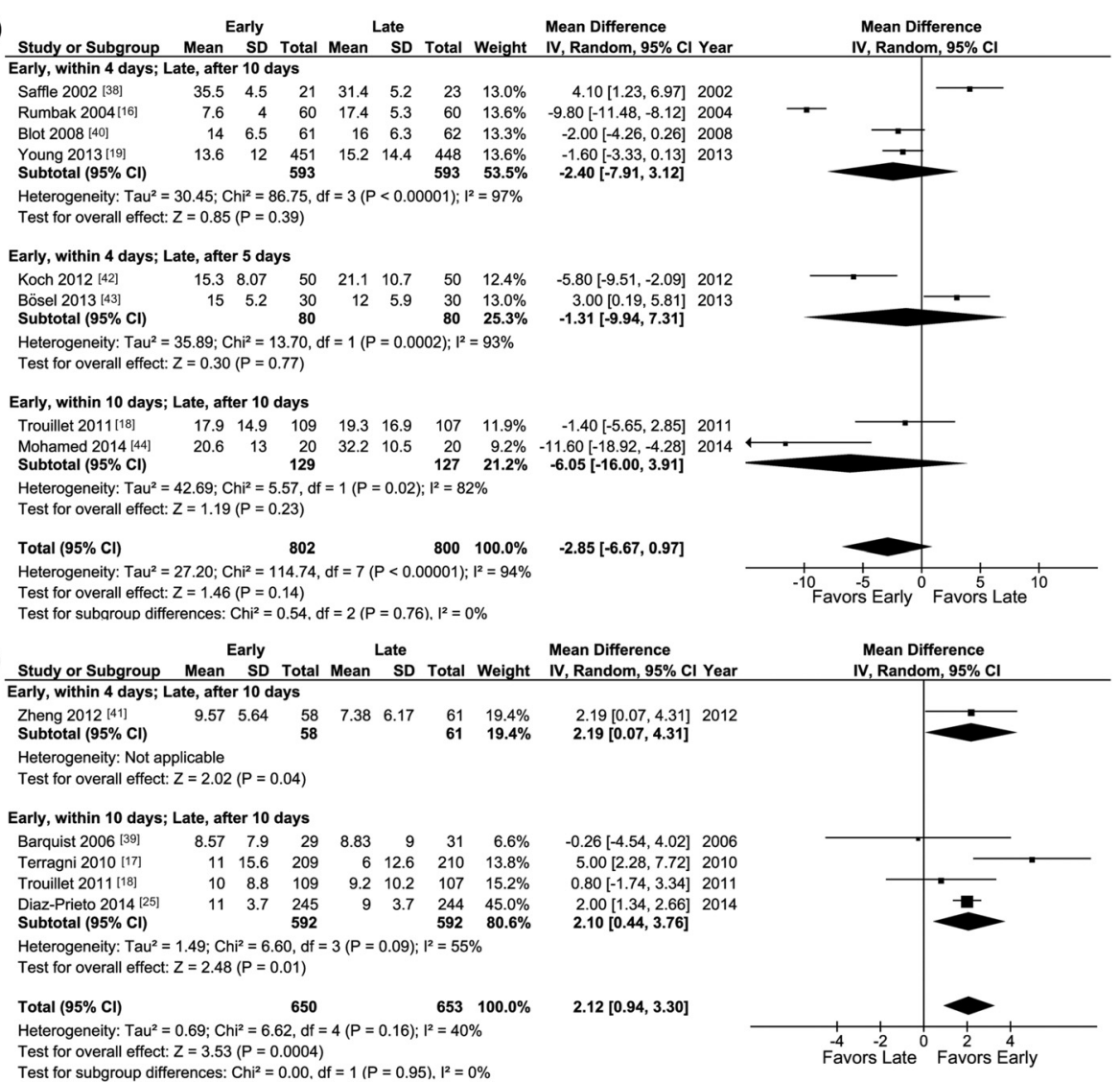

Fig. 2 a Duration of mechanical ventilation. Meta-analysis of the eight studies providing this information. b Ventilator-free days. Meta-analysis of the five studies providing this information. I-V inverse variance

not differ in any of the predefined groups of studies, or overall (OR $0.69(0.45,1.06), p=0.09 ; I^{2} 60 \%, p$ heterogeneity <0.01; Additional file 1: Figure S4).

\section{Mortality}

Short-term ( $\leq 2$ months) mortality rates were reported in 11 studies [16-19, 25, 38-43] (Table 2) and did not differ in any of the predefined groups of studies or overall (OR $0.74(0.55,1.00), p=0.05$; $I^{2} 48 \%, p$ heterogeneity $=0.04$; Fig. 4a). Long-term (>2 months) mortality rates were reported in seven studies [17-19, 25, 42-44] and did not differ in any of the predefined groups of studies but were significantly lower with early than with late tracheotomy overall (OR $0.83(0.69,0.99), p=0.04 ; I^{2} 0 \%, p$ heterogeneity $=0.45$; Fig. $4 \mathrm{~b})$.

\section{Discussion}

Our analysis indicated that early (versus late) tracheotomy was associated with a larger number of VFDs, shorter ICU stay, shorter duration of sedation and lower long-term mortality rates.
Our meta-analysis included a recently published study [25] and gathered a larger number of patients than other recent systematic reviews [20-24], thus improving the ability of the analysis to show differences in major outcomes. Pooled outcome data from most of these reviews did not show a significant reduction in mortality [20, 21, 23, 24], rates of pneumonia [20-24] or duration of mechanical ventilation [20-24] with early compared to late tracheotomy, but one meta-analysis did show significantly reduced long-term mortality [22]. The inclusion of the large study by Diaz-Prieto et al. [25], which included about 500 ICU patients, enabled us to highlight some interesting new differences in outcomes between these two groups of patients. We used robust statistical analysis, including a random-effects model in which the weights of small and large studies are taken into account.

The definition of early and late in previous systematic reviews was before versus after 1 week [23] or 10 days [20-22, 24]. Our broader definitions enabled us to include the study by Koch et al., in which very 


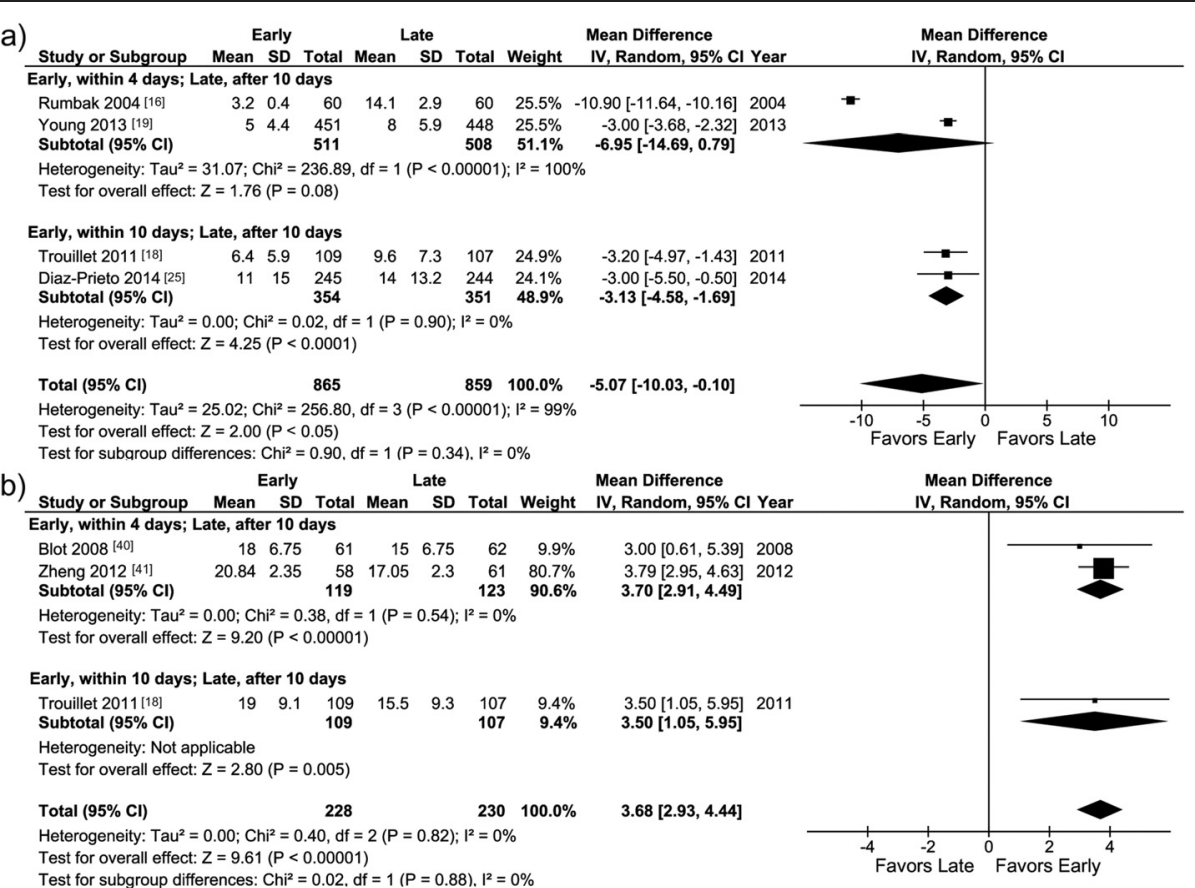

Fig. 3 a Duration of sedation. Meta-analysis of the four studies providing this information. $\mathbf{b}$ Sedation-free days. Meta-analysis of the three studies providing this information. $I-V$ inverse variance

early (within 4 days) tracheotomy was compared to relatively early (after 5 days) tracheotomy [42]. We also included the study by Bösel et al., who compared very early tracheotomy (within 1-3 days after intubation) to what the authors called "standard" timing (between days 7 and 14) [43]. These studies would have been excluded if we had limited the late tracheotomy group to more than 7 or 10 days. Moreover, our cutoffs for the timing of tracheotomy produced some interesting findings in the differences between very early and moderately early procedures.

Tracheotomy rates were generally lower in the late tracheotomy than in the early tracheotomy groups, likely because patients will have recovered or died by the later time point. In addition, there is no reliable means of predicting the likely length of mechanical ventilation. The differences in tracheotomy rates between the early and late group were much larger in the predefined group of studies comparing within 4 days versus after 10 days than that comparing within 10 versus after 10 days.

Our results showed that early tracheotomy was associated with a larger number of VFDs in the group of studies comparing tracheotomy within 10 versus after 10 days. This seems to contradict the policy that tracheotomy should be delayed until after 14 days [7], but does support several reviews that suggest that the need for tracheotomy should be assessed on a daily basis with a definite decision being taken as early as 4-7 days after endotracheal intubation $[9,45,46]$.

As in previous meta-analyses $[20,21]$, early tracheotomy was associated with a shorter duration of sedation. Some [47-49], but not all [50], retrospective observational studies have also reported that early tracheotomy allows a shorter duration of sedation. These differences may be related to the sedation strategies used in these studies.

Our analysis has several limitations. First, there was marked heterogeneity among studies for some of the outcome measures, likely related to the diverse patient groups and characteristics and the different timings of tracheotomy, which are inherent in all systematic reviews on this topic, and the fact that respiratory management may have changed between 2002 and 2015, the dates of publication of the included studies. Second, early tracheotomy may be particularly beneficial in selected groups of patients, such as those with head or spinal cord injury or massive stroke [6, 51], but our meta-analysis could not address this question. Third, adverse effects and cost-effectiveness were not assessed. Finally, the statistical plan included the estimation of WMD using approximate SD values calculated from the IQR. 
a)

$\begin{array}{ccc}\text { Early } & \text { Late } & \begin{array}{c}\text { Odds Ratio } \\ \text { Events Total Events }\end{array} \\ \text { Study or Subgroul Weight } & \text { IV, Random, } 95 \% \mathrm{Cl} \text { Year }\end{array}$ Early, within 4 days; Late, after 10 days Saffle 2002 [38] Rumbak 2004 [16 Blot $2008[40]$ Zheng 2012[41] Young 2013 [19] Subtotal $(95 \% \mathrm{CI})$
Total events

$\begin{array}{ll}17 & 21 \\ 19 & 60 \\ 12 & 61\end{array}$

$\begin{array}{ll}\text { Total events } & 195 \\ \text { Heterogeneity: } \operatorname{Tau}^{2}=0.22 ; \mathrm{Chi}^{2} & =10\end{array}$

\begin{tabular}{lll}
15 & 60 & $9.5 \%$ \\
\hline & $8.1 \%$
\end{tabular}

$\begin{array}{rrr}8 & 58 \\ 139 & 451\end{array}$

$651 \quad 216$

Test for overall effect: $Z=0.82(P=0.41)$

Early, within 4 days; Late, after 5 days

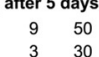
Subtotal (95\% C Total events 80

Heterogeneity: $\mathrm{Tau}^{2}=2.39 ; \mathrm{Chi}^{2}=6.93, \mathrm{df}=1(\mathrm{P}=0.008) ;\left.\right|^{2}=86 \%$ Test for overall effect: $Z=0.71(P=0.48)$

Early, within 10 days; Late, after 10 days Terragni 2010 [17] Trouillet $2011^{[18]}$ Diaz-Prieto 2014 [25] Subtotal $(95 \% \mathrm{CI})$ $\begin{array}{lccc}\text { Total events } & 116 & 141 \\ \text { Heterogeneity: } \text { Tau }^{2}=0.00 ; \mathrm{Chi}^{2}=1.01, \mathrm{df}=3(P=0.80) ; \mathrm{I}^{2}=0 \%\end{array}$ Test for overall effect: $Z=1.76(P=0.08)$

$$
\begin{array}{llllll}
\text { Total }(95 \% \mathrm{Cl}) & \multicolumn{1}{c}{1323} & 1326 & 100.0 \% & 0.74[0.55,1.00]
\end{array}
$$

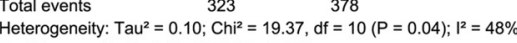
Test for overall effect: $Z=1.95(P=0.05)$

Test for subaroup differences: $\mathrm{Ch}^{2}=0.25$, df $=2(P=0.88), \mathrm{l}^{2}=0 \%$

$1.50[0.36,6.28] 2002$ $0.29[0.14,0.61] 2004$ $0.77[0.33,1.81] 2008$ $1.47[0.48,4.52] 2012$ $0.97[0.73,1.29] 2013$

b)

$\begin{array}{ccc}\text { Early } & \text { Late } & \text { Odds Ratio } \\ \text { Study or Subgroup } & \text { Events Total Events Total Weight } & \text { IV, Random, } 95 \% \mathrm{Cl} \text { Year }\end{array}$ Early, within 4 days; Late, after 10 days Young 2013 [19] $\quad 207 \quad 451$ Subtotal $(95 \% \mathrm{C})$

Total events $207^{451} 217$

Heterogeneity: Not applicable

Test for overall effect: $Z=0.76(P=0.45)$

Early, within 4 days; Late, after 5 days

$\begin{array}{lllllll}\text { Koch } 2012[42] & 10 & 50 & 11 & 50 & 3.6 \%\end{array}$ Bösel $2013[43$

Subtotal $(95 \% \mathrm{Cl})$

$\begin{array}{rr}10 & 50 \\ 8 & 30 \\ & \mathbf{8 0}\end{array}$

$\begin{array}{llll}30 & 18 & 30 & 2.8 \% \\ 80 & & 80 & 6.4 \%\end{array}$

$\begin{array}{lcc}\text { Total events } & 18 & 29 \\ \text { Heterogeneity: } \mathrm{Tau}^{2}=0.56 ; \mathrm{Chi}^{2}=3.05, \mathrm{df}=1(\mathrm{P}=0.08) ; \mathrm{l}^{2}=67 \%\end{array}$

Test for overall effect: $Z=1.15(P=0.25)$

Early, within 10 days; Late, after 10 days

Trouillet $20111^{[18]}$

Diaz-Prieto 2014 [25]

Mohamed 2014 [44]

Subtotal $(95 \% \mathrm{Cl})$

Total events

$\begin{array}{lllll}72 & 144 & 75 & 138 & 15.3 \%\end{array}$

$12 \quad 74$

$\begin{array}{llll}67 \quad 245 & 78 \quad 244 & 22.2 \%\end{array}$

$\begin{array}{lllll}8 & 20 & 8 & 20 & 21 \%\end{array}$ 178

$\begin{array}{rr}20 & 2.1 \% \\ 476 & 44.6 \%\end{array}$

Heterogeneity: $\mathrm{Tau}^{2}=0.00 ; \mathrm{Chi}^{2}=0.41, \mathrm{df}=3(\mathrm{P}=0.94) ; \mathrm{I}^{2}=0 \%$

Test for overall effect: $Z=1.56(P=0.12)$

\begin{tabular}{llllll} 
Total $(95 \% \mathrm{Cl})$ & \multicolumn{1}{c}{1014} & 1004 & $100.0 \%$ \\
Total events & 384 & 424 & &
\end{tabular}

Heterogeneity: $\mathrm{Tau}^{2}=0.00 ; \mathrm{Chi}^{2}=5.77, \mathrm{df}=6(\mathrm{P}=0.45) ; \mathrm{l}^{2}=0 \%$

Test for overall effect: $Z=2.05(P=0.04)$

Test for subgroup differences: $\mathrm{Chi}^{2}=1.16, \mathrm{df}=2(\mathrm{P}=0.56), \mathrm{I}^{2}=0 \%$

$0.39[0.07,2.16] 2006$ $0.78[0.51,1.19] 2010$ $0.67[0.34,1.35] 2011$ $0.87[0.55,1.37] 201$

4

Odds Ratio 


\section{Additional file}

Additional file 1: Table S1. Electronic database search strategy and results. Fig. S1 Preferred reporting items for systematic reviews and meta-analyses (PRISMA) study flow chart. Fig. S2 The quality assessment of included studies. Fig. S3 a Duration of ICU stay. Meta-analysis of the seven studies providing this information. b ICU-free days. Meta-analysis of the three studies providing this information. $\mathrm{Cl}$ confidence interval, $\mathrm{I}-\mathrm{V}$ inverse variance, SD standard deviation. Fig. S4 The incidence of acquired pneumonia. Meta-analysis of the 10 studies providing this information. (DOCX 1069 kb)

\section{Abbreviations}

ADL: activities of daily living; AIS: acute ischemic stroke; APACHE: acute physiology and chronic health evaluation; CENTRAL: Cochrane Central Register of Controlled Trials; Cl: confidence interval; COPD: chronic obstructive pulmonary disease; CPIS: clinical pulmonary infection score; CVA: cerebrovascular accident; GCS: Glasgow coma scale; ICH: intracerebral hemorrhage; ICU: intensive care unit; IQR: interquartile range; MV: mechanical ventilation; OR: odds ratio; $\mathrm{PaO}_{2} / \mathrm{FiO}_{2}$ : partial pressure arterial oxygen/fraction of inspired oxygen; PRISMA: preferred reporting items for systematic reviews and meta-analyses; PTSD: posttraumatic stress disorder; RCT: randomized controlled trial; SAH: subarachnoid hemorrhage; SAPS: simplified acute physiology score; SD: standard deviation; SOFA: sequential organ failure assessment; VFD: ventilator-free day; WMD: weighted mean difference.

\section{Competing interests}

The authors declare that they have no competing interests.

\section{Authors' contributions}

$\mathrm{KH}$ had full access to all of the data in the study and takes responsibility for the integrity of the data and the accuracy of the data analysis. $\mathrm{KH}$ contributed to the study concept and design, data acquisition, data analysis and interpretation, drafting of the manuscript, critical revision of the manuscript for important intellectual content and final approval of the manuscript. MN and ME contributed to the study concept and design, data acquisition and interpretation, and revision and final approval of the manuscript. J-LV contributed to the study concept and design, data analysis and interpretation, critical revision of the manuscript for important intellectual content and final approval of the manuscript. Funding was from institutional funds only.

\section{Author details}

${ }^{1}$ Department of Intensive Care, Erasme University Hospital, Université Libre de Bruxelles, Route de Lennik 808, 1070 Brussels, Belgium. '2Department of Emergency and Critical Care Medicine, Tokushima University Hospital, Tokushima, Japan. ${ }^{3}$ Department Intensive Care, Kobe University Hospital, Kobe-city, Hyogo, Japan.

Received: 7 August 2015 Accepted: 17 November 2015 Published online: 04 December 2015

\section{References}

1. West JB. The physiological challenges of the 1952 Copenhagen poliomyelitis epidemic and a renaissance in clinical respiratory physiology. J Appl Physiol. 2005;99(2):424-32.

2. Severinghaus JW, Astrup P, Murray JF. Blood gas analysis and critical care medicine. Am J Respir Crit Care Med. 1998;157(4 Pt 2):S114-122.

3. Heffner JE. Medical indications for tracheotomy. Chest. 1989;96(1):186-90.

4. Diehl $\mathrm{J}$, El Atrous S, Touchard D, Lemaire F, Brochard L. Changes in the work of breathing induced by tracheotomy in ventilator-dependent patients. Am J Respir Crit Care Med. 1999;159(2):383-8.

5. Davis Jr K, Campbell RS, Johannigman JA, Valente JF, Branson RD. Changes in respiratory mechanics after tracheostomy. Arch Surg. 1999;134(1):59-62.

6. Cheung NH, Napolitano LM. Tracheostomy: epidemiology, indications, timing, technique, and outcomes. Respir Care. 2014;59(6):895-915.

7. Freeman BD, Morris PE. Tracheostomy practice in adults with acute respiratory failure. Crit Care Med. 2012;40(10):2890-6.

8. Heffner JE, Miller KS, Sahn SA. Tracheostomy in the intensive care unit. Part 1: Indications, technique, management. Chest. 1986;90(2):269-74.
9. Rana S, Pendem S, Pogodzinski MS, Hubmayr RD, Gajic O. Tracheostomy in critically ill patients. Mayo Clin Proc. 2005;80(12):1632-8.

10. Epstein SK. Late complications of tracheostomy. Respir Care. 2005;50(4):542-9.

11. Stauffer JL, Olson DE, Petty TL. Complications and consequences of endotracheal intubation and tracheotomy. A prospective study of 150 critically ill adult patients. Am J Med. 1981;70(1):65-76.

12. Plummer AL, Gracey DR. Consensus conference on artificial airways in patients receiving mechanical ventilation. Chest. 1989;96(1):178-80.

13. Brook $A D$, Sherman $G$, Malen J, Kollef MH. Early versus late tracheostomy in patients who require prolonged mechanical ventilation. Am J Crit Care. 2000;9(5):352-9.

14. Arabi Y, Haddad S, Shirawi N, Al SA. Early tracheostomy in intensive care trauma patients improves resource utilization: a cohort study and literature review. Crit Care. 2004;8(5):R347-52.

15. Griffiths J, Barber VS, Morgan L, Young JD. Systematic review and meta-analysis of studies of the timing of tracheostomy in adult patients undergoing artificial ventilation. BMJ. 2005;330(7502):1243.

16. Rumbak MJ, Newton M, Truncale T, Schwartz SW, Adams JW, Hazard PB. A prospective, randomized, study comparing early percutaneous dilational tracheotomy to prolonged translaryngeal intubation (delayed tracheotomy) in critically ill medical patients. Crit Care Med. 2004;32(8):1689-94.

17. Terragni PP, Antonelli M, Fumagalli R, Faggiano C, Berardino M, Pallavicini $F B$, et al. Early vs late tracheotomy for prevention of pneumonia in mechanically ventilated adult ICU patients: a randomized controlled trial. JAMA. 2010;303(15):1483-9.

18. Trouillet JL, Luyt CE, Guiguet M, Ouattara A, Vaissier E, Makri R, et al. Early percutaneous tracheotomy versus prolonged intubation of mechanically ventilated patients after cardiac surgery: a randomized trial. Ann Intern Med. 2011;154(6):373-83.

19. Young D, Harrison DA, Cuthbertson BH, Rowan K, TracMan Collaborators Effect of early vs late tracheostomy placement on survival in patients receiving mechanical ventilation: the TracMan randomized trial. JAMA. 2013; 309(20):2121-9.

20. Meng L, Wang CM, Li JX, Zhang J. Early versus late tracheostomy in critically ill patients: a systematic review and meta-analysis. Clin Respir J. 2015. doi:10.1111/crj.12286.

21. Szakmany $T$, Russell $P$, Wilkes AR, Hall JE. Effect of early tracheostomy on resource utilization and clinical outcomes in critically ill patients: meta-analysis of randomized controlled trials. $\mathrm{Br} J$ Anaesth. 2015;114(3):396-405

22. Andriolo BN, Andriolo RB, Saconato H, Atallah ÁN, Valente O. Early versus late tracheostomy for critically ill patients. Cochrane Database Syst Rev. 2015;1, CD007271.

23. Siempos II, Ntaidou TK, Filippidis FT, Choi AM. Effect of early versus late or no tracheostomy on mortality and pneumonia of critically ill patients receiving mechanical ventilation: a systematic review and meta-analysis. Lancet Respir Med. 2015;3(2):150-8.

24. Huang H, Li Y, Ariani F, Chen X, Lin J. Timing of tracheostomy in critically il patients: a meta-analysis. PLoS One. 2014;9(3), e92981.

25. Diaz-Prieto A, Mateu A, Gorriz M, Ortiga B, Truchero C, Sampietro N, et al. A randomized clinical trial for the timing of tracheotomy in critically ill patients: factors precluding inclusion in a single center study. Crit Care. 2014;18(5):585

26. Liberati A, Altman DG, Tetzlaff J, Mulrow C, Gøtzsche PC, loannidis JP, et al The PRISMA statement for reporting systematic reviews and meta-analyses of studies that evaluate health care interventions: explanation and elaboration. PLoS Med. 2009;6(7), e1000100.

27. Higgins J, Green S. Cochrane Handbook for Systematic Reviews of Interventions (Ver. 5.1.0.). 2011.

28. Hozo SP, Djulbegovic B, Hozo I. Estimating the mean and variance from the median, range, and the size of a sample. BMC Med Res Methodol. 2005;5:13.

29. Dunham CM, LaMonica C. Prolonged tracheal intubation in the trauma patient. J Trauma. 1984;24(2):120-4

30. El-Naggar M, Sadagopan S, Levine H, Kantor H, Collins VJ. Factors influencing choice between tracheostomy and prolonged translaryngeal intubation in acute respiratory failure: a prospective study. Anesth Analg. 1976;55(2):195-201.

31. Rodriguez JL, Steinberg SM, Luchetti FA, Gibbons KJ, Taheri PA, Flint LM. Early tracheostomy for primary airway management in the surgical critical care setting. Surgery. 1990;108(4):655-9. 
32. Sugerman HJ, Wolfe L, Pasquale MD, Rogers FB, O'Malley KF, Knudson M, et al. Multicenter, randomized, prospective trial of early tracheostomy. J Trauma. 1997;43(5):741-7

33. Bouderka MA, Fakhir B, Bouaggad A, Hmamouchi B, Hamoudi D, Harti A. Early tracheostomy versus prolonged endotracheal intubation in severe head injury. J Trauma. 2004;57(2):251-4.

34. Dunham CM, Cutrona AF, Gruber BS, Calderon JE, Ransom KJ, Flowers LL. Early tracheostomy in severe traumatic brain injury: evidence for decreased mechanical ventilation and increased hospital mortality. Int J Burns Trauma. 2014:4(1):14-24.

35. Bylappa K, Mohiyudin A, Delphine W, Silvia CR, Krishnamurthy D, Pyarajan MS. A comparative study of early and late tracheostomy in patients requiring prolonged tracheal intubation. World Articles Ear Nose Throat. 2011;4(2). http://www.waent.org/archives/2011/Nol4-2/20111215Tracheostomy-Intubation/late-tracheotomy.htm

36. Sabouri M, Esmaili TM, Hosseini B. The effects of early tracheostomy on outcomes of patients with severe head injury. J Isfahan Med School. 2009;27(95):211-6

37. Lu W, Xia ZF, Chen YL. Clinical study on the comparison of prophylactic tracheotomy with emergent tracheotomy after inhalation injury. Chin J Burns (Zhonghua shao shang za zhi). 2003;19(4):233-5.

38. Saffle JR, Morris SE, Edelman L. Early tracheostomy does not improve outcome in burn patients. J Burn Care Rehabil. 2002;23(6):431-8.

39. Barquist ES, Amortegui J, Hallal A, Giannotti G, Whinney R, Alzamel H, et al. Tracheostomy in ventilator dependent trauma patients: a prospective, randomized intention-to-treat study. J Trauma. 2006:60(1):91-7.

40. Blot F, Similowski T, Trouillet $J$, Chardon P, Korach JM, Costa MA, et al. Early tracheotomy versus prolonged endotracheal intubation in unselected severely ill ICU patients. Intensive Care Med. 2008;34(10):1779-87.

41. Zheng Y, Sui F, Chen XK, Zhang GC, Wang XW, Zhao S, et al. Early versus late percutaneous dilational tracheostomy in critically ill patients anticipated requiring prolonged mechanical ventilation. Chin Med J. 2012;125(11):1925-30.

42. Koch T, Hecker B, Hecker A, Brenck F, Preuß M, Schmelzer T, et al. Early tracheostomy decreases ventilation time but has no impact on mortality of intensive care patients: a randomized study. Langenbecks Arch Surg. 2012;397(6):1001-8.

43. Bösel J, Schiller P, Hook Y, Andes M, Neumann JO, Poli S, et al. Strokerelated early tracheostomy versus prolonged orotracheal intubation in neurocritical care trial (SETPOINT): a randomized pilot trial. Stroke. 2013:44(1):21-8.

44. Mohamed KAE, Mousa AY, ElSawy AS, Saleem AM. Early versus late percutaneous tracheostomy in critically ill adult mechanically ventilated patients. Egyp J Chest Dis Tubercu. 2014;63(2):443-8.

45. Bittner EA, Schmidt UH. The ventilator liberation process: update on technique, timing, and termination of tracheostomy. Respir Care. 2012;57(10):1626-34.

46. King C, Moores LK. Controversies in mechanical ventilation: when should a tracheotomy be placed? Clin Chest Med. 2008;29(2):253-63.

47. Gandía-Martínez F, Martínez-Gil I, Andaluz-Ojeda D, Bobillo de Lamo F, Parra-Morais L, Díez-Gutiérrez F, et al. Analysis of early tracheostomy and its impact on development of pneumonia, use of resources and mortality in neurocritically ill patients. Neurocirugia. 2010;21(3):211-21.

48. Aissaoui Y, Azendour H, Balkhi H, Haimeur C, Kamili Drissi N, Atmani M. Timing of tracheostomy and outcome of patients requiring mechanical ventilation. Ann Fr Anesth Reanim. 2007;26(6):496-501.

49. Veelo DP, Dongelmans DA, Binnekade JM, Korevaar JC, Vroom MB, Schultz MJ. Tracheotomy does not affect reducing sedation requirements of patients in intensive care-a retrospective study. Crit Care. 2006;10(4):R99.

50. Nieszkowska A, Combes A, Luyt CE, Ksibi H, Trouillet JL, Gibert C, et al. Impact of tracheotomy on sedative administration, sedation level, and comfort of mechanically ventilated intensive care unit patients. Crit Care Med. 2005;33(11):2527-33.

51. Scales DC, Thiruchelvam D, Kiss A, Redelmeier DA. The effect of tracheostomy timing during critical illness on long-term survival. Crit Care Med. 2008;36(9):2547-57.

\section{Submit your next manuscript to BioMed Central and we will help you at every step:}

- We accept pre-submission inquiries

- Our selector tool helps you to find the most relevant journal

- We provide round the clock customer support

- Convenient online submission

- Thorough peer review

- Inclusion in PubMed and all major indexing services

- Maximum visibility for your research

Submit your manuscript at www.biomedcentral.com/submit 